\title{
АДАПТИВНАЯ ИНТЕРПРЕТАЦИЯ РЕЗУЛЬТАТОВ ГИДРОДИНАМИЧЕСКИХ ИССЛЕДОВАНИЙ ГОРИЗОНТАЛЬНЫХ СКВАЖИН НА ПРОГНОЗИРУЮЩИХ МОДЕЛЯХ
}

\author{
Сергеев Виктор Леонидович', \\ SVL00@tpu.ru
}

Донг Ван Хоанг',

hoang.tpu@gmail.com

Фам Динь Ан',

phaman.tpu@gmail.com
Национальный исследовательский Томский политехнический университет, Россия, 634050, г. Томск, пр. Ленина, 30.

Актуальность обусловлена необходимостью интерпретации результатов нестационарных гидродинамических исследований горизонтальных скважин в условиях отсутствия на кривой восстановления давления участка позднего радиального потока. Целью исследования является разработка и исследование моделей и алгоритмов адаптивной идентификации и интерпретации результатов гидродинамических исследований горизонтальных скважин с прогнозированием недовосстановленного участка кривой забойного давления позднего радиального потока.

Методы исследования. Использованы теоретические и практические разработки в области адаптивной интерпретации гидродинамических исследований скважин, методов оптимизации функций, линейной алгебры, современных исследований системного анализа, идентификации и адаптации систем. Для решения задач идентификации и прогноза использованы эволюционные феноменологические модели кривой восстановления забойного давления с переменными, зависящими от времени параметрами, с учетом дополнительной информации о пластовом давлении. Интерпретация кривой восстановления забойного давления осуществлялась на основе метода адаптивной идентификации. Решение задач проводилось с использованием промысловых данных гидродинамических исследований горизонтальных скважин нефтяных месторождений по кривой восстановления давления.

Результаты. Исследованы потенциальные возможности эволюционных феноменологических моделей с переменными параметрами для идентификации и прогноза кривой восстановления забойного давления при отсутствии участка позднего радиального потока. На примерах обработки результатов гидродинамических исследований горизонтальных скважин нефтяного месторождения показано, что разработанные модели и алгоритмы адаптивной идентификации и интерпретации позволяют: прогнозировать забойное давление на недовосстановленном участке кривой восстановления давлении, определять латеральную проницаемость, скин-фактор, эффективную длину скважины и время завершения исследований в процессе их проведения, сократить время простоя скважин.

\section{Ключевые слова:}

Интерпретация, идентификация, адаптация, гидродинамические исследования скважин, кривая восстановления давления, горизонтальные скважины, нефтяные низкопроницаемые коллектора, феноменологические модели, априорная информация.

\section{Введение}

В настоящее время использование традиционных графоаналитических методов интерпретации результатов нестационарных гидродинамических исследований (ГДИ) горизонтальных скважин, основанных на анализе кривой восстановления забойного давления (КВД) и ее производной, вызывает значительные трудности, связанные с отсутствием на КВД участка позднего радиального потока либо со значительными искажениями на этом участке забойного давления. Так, для получения полноценных КВД горизонтальных скважин низкопроницаемых коллекторов, с присутствием участка позднего радиального потока, требуется проведение достаточно продолжительных исследований, порядка 500 часов и более. Интерпретация таких КВД требует привлечения квалифицированных интерпретаторов, снижает оперативность получения результатов, приводит к простоям скважин и значительным материальным затратам [1-8].
В этой связи актуальной является задача разработки моделей и алгоритмов оперативной обработки результатов ГДИ горизонтальных скважин в процессе проведения гидродинамических исследований в условиях частичного либо полного отсутствия на КВД участка позднего радиального потока длительностью порядка 50-100 часов, что является более приемлемым с точки зрения простоя скважин. Решение данной задачи было рассмотрено в работе [9], где предложен метод адаптивной интерпретации ГДИ горизонтальных скважин нефтяных пластов с использованием уравнения Вольтерра 1-го рода с переменными параметрами. Показано, что разработанные модели и алгоритмы идентификации и интерпретации позволяют оперативно обрабатывать короткие недовосстановленные КВД, поскольку стабилизация оценок латеральной проницаемости наступает уже на стадии линейного потока либо на начальной стадии позднего радиального потока. Однако, если на стадии линейного потока стабилизация оценок латераль- 
ной проницаемости не наблюдается и требуется по ряду причин прервать исследования, вопрос идентификации и интерпретации таких недовосстановленных КВД остается открытым.

В данной работе предлагается метод оперативной идентификации и интерпретации результатов ГДИ горизонтальных скважин на прогнозирующих феноменологических моделях, позволяющий определять забойное давление на недовосстановленном участке КВД, пластовое давление и фильтрационные параметры пласта в условиях отсутствия позднего радиального потока, когда стабилизация оценок латеральной проницаемости не наступает.

\section{Модели и алгоритмы идентификации и прогноза КВД}

Основой алгоритмов идентификации и прогноза недовосстановленного участка позднего радиального потока КВД являются феноменологические модели забойного давления с переменными параметрами $\alpha_{n}=a\left(t_{n}\right)$, с учетом дополнительной информации о пластовом давлении $\bar{p}_{\text {пл }}$ вида:

$$
\left\{\begin{array}{l}
P_{3}^{*}\left(t_{n}\right)=P_{3}\left(t_{0}\right)+f\left(t_{n}, \alpha_{n}\right)+\xi\left(t_{n}\right), n=\overline{1, n k}, \\
h_{\text {к }, n} \bar{p}_{\text {пл }}=f\left(\bar{t}_{\mathrm{B}}, \alpha_{n}\right)+\eta_{n} .
\end{array}\right.
$$

где $P_{3}^{*}\left(t_{n}\right), f\left(t_{n}, \alpha_{n}\right)$ - фактические и вычисленные на основе феноменологической модели (табл. 1) значения забойного давления, полученные в разные моменты времени $t_{n} \in\left[t_{0}, t_{n k}\right] ; t_{0}$ - время начала исследования, ч; $t_{n k}-$ момент времени завершения исследования, ч; $P_{3}\left(t_{0}\right)$ - значение забойного давления в момент времени $t_{0}$, атм; $\alpha_{n}(n=\overline{1, n k})$ - параметры феноменологической модели забойного давления в моменты времени $t_{n} ; \bar{P}_{\text {пл }}-$ экспертная оценка пластового давления, атм; $\bar{t}_{\mathrm{B}}-$ экспертная оценка момента времени восстановления забойного давления до пластового, ч; $h_{\mathrm{k}, n}$ - параметр корректировки экспертной оценки пластового давления; $\xi_{n}, \eta_{n}$ - случайные величины, представляющие погрешности измерений забойного давления, ошибки экспертных оценок, и т. п.

Прогнозные значения недовосстановленного участка КВД на время $\tau$ вычисляем по формуле (2).

$$
\widehat{P}_{3}\left(t_{n}+\tau\right)=P_{3}\left(t_{0}\right)+f\left(t_{n}+\tau, \alpha_{n}^{*}\left(h_{n}^{*}\right)\right),
$$

где оценки параметров модели (1) $\alpha_{n}^{*}\left(h_{n}\right)$ и управляющих параметров $h_{n}^{*}$ определяем согласно методу адаптивной интерпретации путем решения оптимизационных задач [10]

$$
\begin{gathered}
\alpha_{n}^{*}\left(h_{n}\right)=\arg \min _{\alpha_{n}}\left(J_{0}\left(\alpha_{n}, h_{\varsigma n}\right)+J_{a}\left(\alpha_{n}, h_{p \eta}, h_{\text {ки }}\right)\right), \\
h_{n}^{*}=\arg \min _{h_{n}} J_{0}\left(\alpha_{n}^{*}\left(h_{n}\right)\right),
\end{gathered}
$$

где запись $\arg \min _{x} f(x)$ означает точку минимума $x^{*}$ функции $f(x)\left(f\left(x^{*}\right)=\min _{x} f(x)\right)$;

$$
\begin{gathered}
J_{0}\left(\alpha_{n}, h_{3, n}\right)= \\
=\sum_{i=1}^{n} \mathbf{K}\left(\frac{t_{n}-t_{i}}{h_{3, n}}\right)\left(P_{3}^{*}\left(t_{n}\right)-\left(P_{3}\left(t_{0}\right)+f\left(t_{n}, \alpha_{n}\right)\right)\right)^{2}
\end{gathered}
$$

- показатель качества модели забойного давления;

$$
J_{a}\left(\alpha_{n}, h_{\delta, n}, h_{\mathrm{\kappa}, n}\right)=h_{p, n}\left(h_{k, n} \bar{p}_{\text {пл }}-f\left(\bar{t}_{\mathrm{в}}, \alpha_{n}\right)\right)^{2}
$$

- показатель качества модели экспертной оценки пластового давления $\bar{P}_{\text {пл }} ; h_{n}^{*}\left(h_{3, n}, h_{p, n}, h_{\mathrm{k}, n}\right)-$ вектор управляющих параметров, забывания, регуляризации и корректировки экспертной оценки пластового давления, соответственно [11].

На рис. 1, 2 приведены исходные КВД (линия 1) скважин № 1, 2 Верхнечонского месторождения и восстановленные значения забойного давления (линия 2), полученные с использованием феноменологической модели № 3 (табл. 1).

В табл. 1 приведены феноменологические модели КВД [12-19] и относительные ошибки $\delta_{\tau}(5)$ прогноза забойного давления на время $\tau$, полученные с момента времени завершения линейного потока $t_{\text {л }}=10$ ч (для скважины № 1) и $t_{\text {л }}=40$ ч (для скважины № 2) с использованием промысловых данных забойного давления, приведенных на рис. 1, 2.

$$
\delta_{\tau}=\left|\frac{P_{3}\left(t_{n}+\tau\right)-\widehat{P}_{3}\left(t_{n}+\tau\right)}{P_{3}\left(t_{n}+\tau\right)}\right| \cdot 100,
$$

где $P_{3}\left(t_{n}+\tau\right)$ - значения забойных давлений, атм; $\hat{P}_{3}\left(t_{n}+\tau\right)-$ прогнозные значения забойного давления, атм.

На рис. 3, 4 приведены оценки пластового давления $P_{\text {пл }}^{*}$ и их относительные ошибки $\delta_{\text {пл }}$, определенные по формулам (6) и (7).

$$
\begin{gathered}
P_{\text {пл }}^{*}=f\left(\bar{t}_{\text {в }}, \alpha_{n}^{*}\left(h_{n}^{*}\right)\right) ; \\
\delta_{\text {пл }}=\left|\frac{P_{\text {пл }}-P_{\text {пл }}^{*}}{P_{\text {пл }}}\right|
\end{gathered}
$$

с использованием феноменологической модели № 3, где $P_{\text {пл }}$ - точное значение пластового давления скважины № 2 , атм.

Отметим, что точное значение пластового давления скважины № $2 P_{\text {пл }}=106,7$ атм, а экспертная оценка пластового давления $\bar{P}_{\text {пл }}$ принималась равной 111 атм, с ошибкой порядка $4 \%$.

Решение оптимизационных задач по определению параметров модели КВД (3) проводилось с использованием метода деформированного многогранника [20]. Оптимизационная задача по определению вектора управляющих параметров (4), по аналогии с [11], сводилась к последовательному решению одномерных оптимизационных задач по определению параметра регуляризации, корректировки и забывания методом дихотомии [20].

На рис. 3, 4 приведены оценки пластового давления и их относительные ошибки, полученные с использованием феноменологических моделей КВД, представленных в табл. 1. 
Таблииа 1. Феноменологические модели КВД и относительнал ошибка прогноза забойного давления

Table 1. Phenomenological models and relative errors of forecas ting of bottomhole pressure

\begin{tabular}{|c|c|c|c|c|c|c|}
\hline \multirow[t]{2}{*}{$\begin{array}{c}\text { Модели КВД } \\
\text { Models of pressure } \\
\text { build-up curve }\end{array}$} & \multirow[t]{2}{*}{$\begin{array}{l}\text { Название } \\
\text { (автор) } \\
\text { Name } \\
\text { (author) }\end{array}$} & \multirow{2}{*}{ 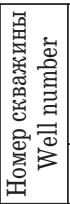 } & \multicolumn{4}{|c|}{$\begin{array}{c}\text { Относительная ошибка } \\
\text { прогноза на время } \tau \text { (ч), \% } \\
\text { Relative errors of forecasting } \\
\text { at the moment } \tau \text { (hour), } \%\end{array}$} \\
\hline & & & $\tau=40$ & $\tau=80$ & $\tau=120$ & $\tau=160$ \\
\hline \multirow{2}{*}{$\begin{array}{l}\Delta Ð=\frac{1}{\left(1+e^{-\alpha_{3} t}\right)} \times \\
\quad \times\left(\alpha_{1}+\alpha_{2} \ln t\right)\end{array}$} & \multirow[b]{2}{*}{$\begin{array}{l}\text { Хоанг-Ан } \\
\text { Hoang-An }\end{array}$} & 1 & 0,02 & & & \\
\hline & & 2 & 0,31 & 0,42 & 0,56 & 0,53 \\
\hline \multirow{2}{*}{$\Delta P=\alpha_{1}\left(1-e^{-\alpha_{2} t}\right)^{\alpha_{3}}$} & \multirow{2}{*}{$\begin{array}{l}\text { Берталанфи } \\
\text { Bertalanffy }\end{array}$} & 1 & 0,065 & 0,027 & 0,058 & 0,12 \\
\hline & & 2 & 0,06 & 0,56 & 0,98 & 0,45 \\
\hline \multirow{2}{*}{$\Delta P=\alpha_{1} e^{\alpha_{2} t^{t^{-\alpha_{3}}}}$} & \multirow{2}{*}{$\begin{array}{l}\text { Кольрауш } \\
\text { Kohlrausch }\end{array}$} & 1 & 0,068 & 0,071 & 0,12 & 0,14 \\
\hline & & 2 & 0,13 & 0,48 & 1,12 & 0,89 \\
\hline
\end{tabular}

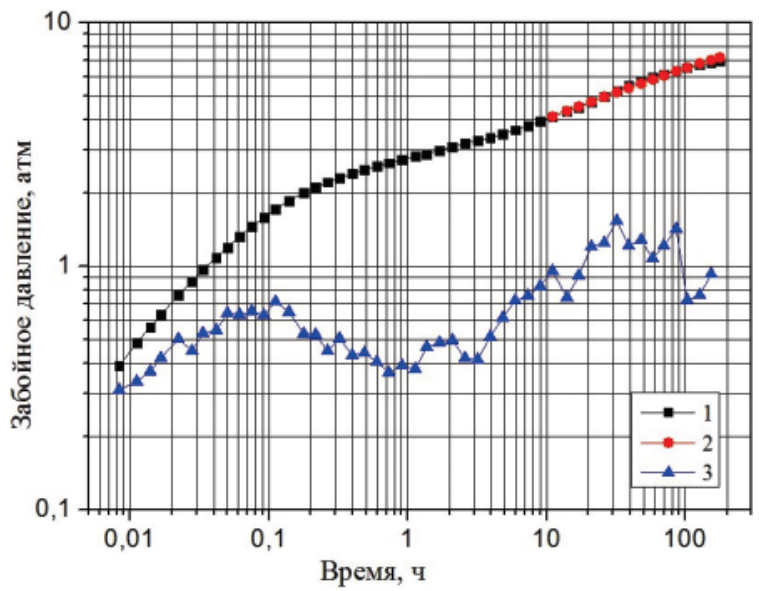

Puc. 1. Исходная КВД скважины № 1 (линия 1) и её производ ная (линия 3), прогнозная (восставленная) КВД (линия 2) с использованием модели Берталанфи

Fig. 1. Initial pressure build-up curve of well no. 1 (line 1) and its pressure derivative, expected (recovered) pressure buildup curves (line 2) using the Bertalanffy model

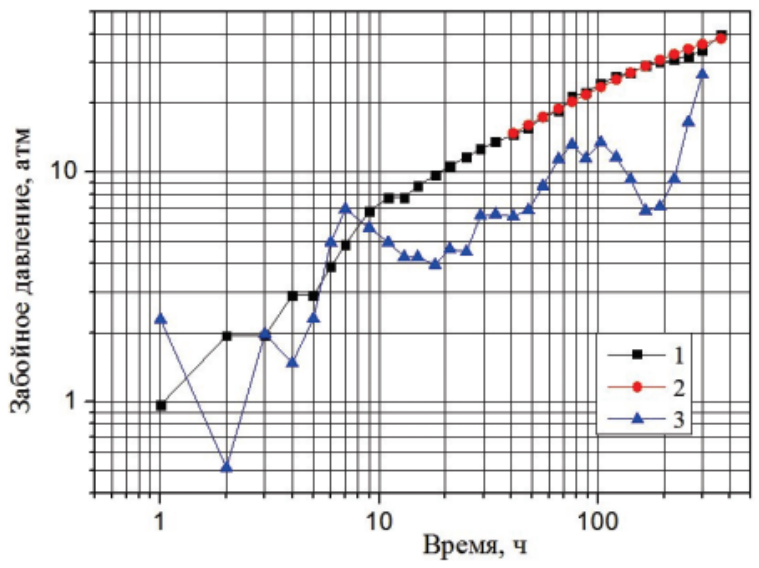

Puc. 2. Исходная КВД скважины № 2 (линия 1) и её производ ная (линия 3), прогнозная (восставленная) КВД (линия 2) с использованием модели Берталанфи

Fig. 2. Initial pressure build-up curve of well no. 2 (line 1) and its pressure derivative, expected (recovered) pressure buildup curves (line 2) using the Bertalanffy model

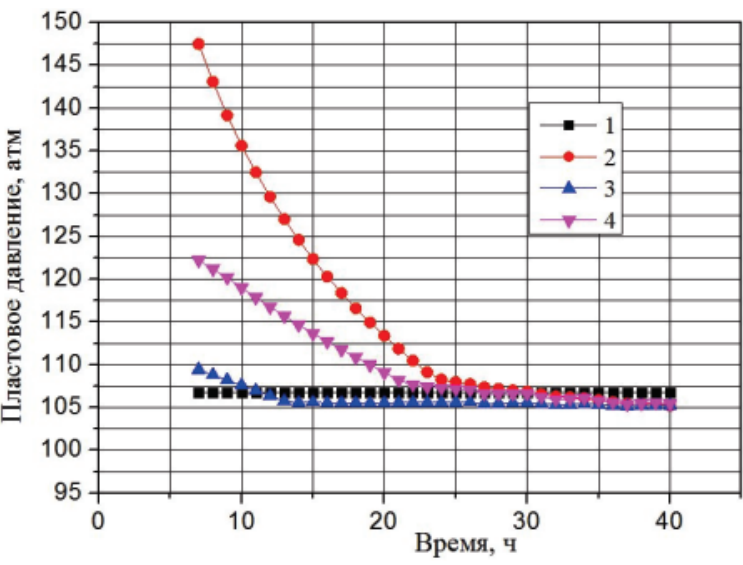

Pис.3. Оиенки пластового давления скважины № 2 (линия 1 фактическое значение пластового давления; линия 2- по модели Хоанг-Ан; линия 3 - по модели Берталанфи; линия 4 - по модели Кольрауш)

Fig. 3. Estimates of reservoir pressure of well no. 2 (line 1 - actual value of reservoir pressure; line 2 - with the Hoang-An model; line 3 - with the Bertalanffy model; line 4 - with the Kohlrausch model)

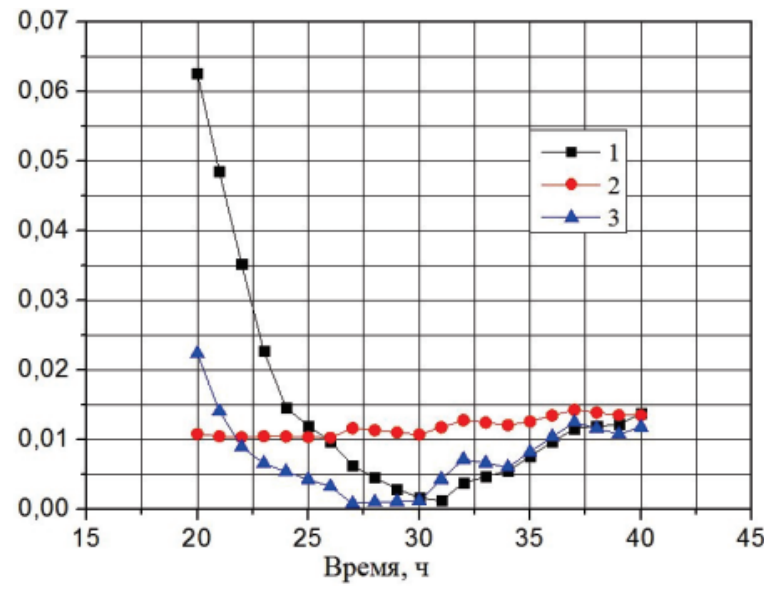

Рис.4. Относительные ошибки оценок пластового давления скважины № 2 (линия 1 - по модели Хоанг-Ан; линия 2 по модели Берталанфи; линия 3 - по модели Кольрауш)

Fig. 4. Relative errors of reservoir pressure estimations of well no. 2 (line 1 - with the Hoang-An model; line 2 - with the Bertalanffy model; line 3 - with the Kohlrausch model)

Анализ результатов, приведенных на рис. 3,4 и в табл. 1, показывает, что алгоритмы адаптивной идентификации и прогноза на основе феноменологических моделей КВД позволяют достаточно точно определять пластовое давление (с ошибкой порядка $0,1-0,2 \%$ ) и прогнозировать забойные давления на завершающей стадии линейного потока.

\section{Результаты интерпретации КВД с восстановлением позднего радиального потока}

Процесс интерпретации КВД с прогнозированием недовосстановленного участка

забойного давления осуществляется по следующей схеме:

1. Определяем латеральную проницаемость пласта, пластовое давление и скин-фактор с ис- 
пользованием модели КВД позднего радиального режима течения [9]. В момент времени стабилизации оценок латеральной проницаемости процесс гидродинамических исследований завершается (рис. 5, 6, линия 2).

2. Если стабилизация оценок латеральной проницаемости не наступает (рис. 5, 6, линия 1), то осуществляется прогноз недовосстановленного участка КВД с использованием прогнозирующих моделей (2).

3. С использованием восстановленных значений забойных давлений и модели КВД (9) определяем оценки латеральной проницаемости до их стабилизации, а также оценки пластового давления, горизонтальной эффективной длины ствола и скин-фактора скважины.

Результаты интерпретации КВД с восстановлением позднего радиального потока горизонтальных скважин № 1, 2 приведены на рис. 5, 6 и в табл. 3. Процесс восстановления забойного давления начинался с момента времени $t_{л}=10$ ч и $t_{л}=40$ ч для скважин № 1,2 соответственно. На рис. 5, 6 приведены оценки латеральной проницаемости скважин № 1, 2 (8).

$$
k_{r, n}^{*}=\frac{C_{s} q_{0} \mu B}{4 \pi \alpha_{1, n}^{*}\left(\beta_{n}^{*}, h_{n}^{*}\right) h}
$$

для полных и восстановленных КВД, полученные с использованием модели забойного давления вида [9]:

$$
\left\{\begin{array}{l}
P_{3, n}^{*}=P_{3, n}+\xi_{n}=P\left(t_{0}\right)+ \\
+\alpha_{1 n} \int_{t_{n l}}^{t_{n k}} \frac{\left(1-q\left(\alpha_{3 n}, t_{n}\right)\right)}{t_{n}-\tau} \exp \left(-\frac{\alpha_{2 n}}{\left(t_{n}-\tau\right)}\right) d \tau+\xi_{n}, \\
n=\overline{n l, n k ;} \\
\bar{p}_{\text {пл }}=P_{3, n+\tau}+\eta_{2, n+\tau}, \tau>t_{k},
\end{array}\right.
$$

где $\quad q\left(\alpha_{3 n}, t_{n}\right) \rightarrow 0$ при $\quad t_{n} \rightarrow \infty . \quad \alpha_{1 n}=\frac{C_{s} q_{0} \mu B}{4 \pi k_{r, n} h}$; $\alpha_{2 n}=\frac{\phi \mu r_{\text {пр,n }}^{2}}{4 k_{r, n}} ; k_{r, n}-$ латеральная проницаемость (проницаемость позднего радиального потока); $q_{0}$ дебит скважины в момент ее остановки при $t_{0}$, $\mathrm{cm}^{3} / \mathrm{c} ; \phi-$ пористость, доля; $\mu$ - вязкость нефти, сПз; $B$ - объемный коэффициент нефти, $\mathrm{M}^{3} / \mathrm{M}^{3}$; $r_{\text {пр, }}$ - приведенный радиус скважины, см; h - толщина пласта, см; $C_{s}$ - константа, зависящая от используемой системы единиц; $\chi_{n}, \eta_{n}$ - случайные неконтролируемые факторы, представляющие различного рода погрешности измерений забойных давлений, ошибки экспертных оценок, неточности модели КВД и т. п.

Для интегрированной модели (9) оптимальные оценки параметров и управляющих параметров определяются по схеме, изложенной в работе [20].

В табл. 2 приведены используемые при интерпретации исходные данные и дополнительные априорные сведения для скважин № 1,2 .

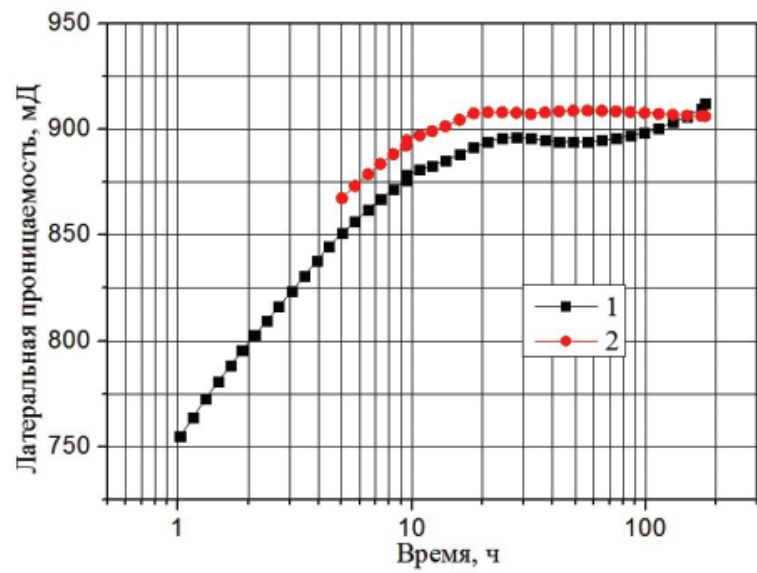

Puс. 5. Оиенки латеральной пронииаелости скважины № 1 (линия 1 - для полной КВД, линия 2 - для восстановленной КВД)

Fig. 5. Estimates of radial permeability of well no. 1 (line 1 - with total pressure build-up curve; line 2 - with recovered pressure build-up curve)

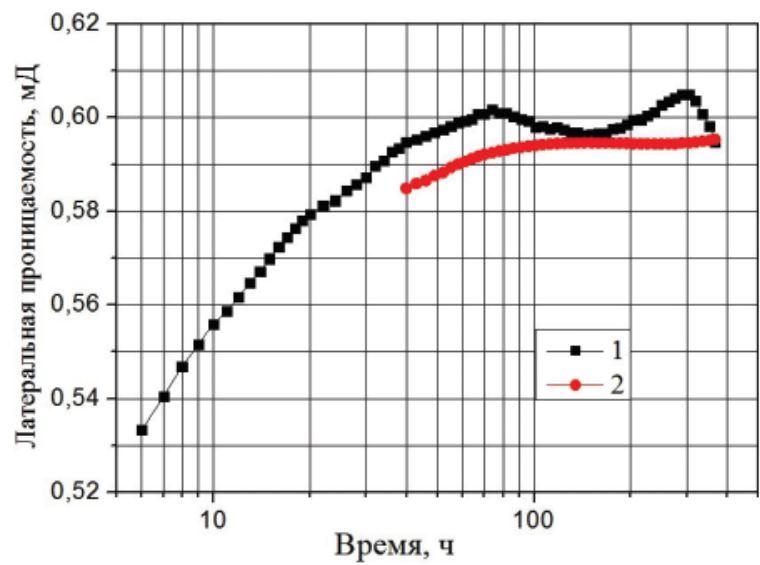

Pис. 6. Оиенки латеральной проницаемости скважины № 2 (линия 1 - для полной КВД, линия 2 - для восстановленной КВД)

Fig. 6. Estimates of radial permeability of well no. 2 (line 1 - with total pressure build-up curve; line 2 - with recovered pressure

\begin{tabular}{|c|c|c|}
\hline \multirow{2}{*}{$\begin{array}{c}\text { Исходные данные и экспертные оценки параметров } \\
\text { пласта и скважин № 1, } 2 \text { (в системе единиц СИ) } \\
\text { Initial data and expert estimates of reservoir and } \\
\text { well parameters (International System of Units (SI)) }\end{array}$} & \multicolumn{2}{|c|}{$\begin{array}{l}\text { Скважина } \\
\text { Well }\end{array}$} \\
\hline & 1 & 2 \\
\hline $\begin{array}{l}\text { Динамическая вязкость нефти, сП } \\
\text { Dynamic viscosity of oil, cP }\end{array}$ & 3,92 & 0,54 \\
\hline $\begin{array}{l}\text { Коэффициент сжимаемости нефти, атм } \\
\text { Oil compressibility factor, } \text { atm }^{-1}\end{array}$ & $1,78 \cdot 10^{-4}$ & $1,95 \cdot 10^{-4}$ \\
\hline Радиус скважины, м/Well radius, m & 0,108 & 0,108 \\
\hline Атмосферное давление, ат/Atmosphere pressure, at & 1,033 & 1,033 \\
\hline $\begin{array}{l}\text { Температура при нормальных условия }\left(+20{ }^{\circ} \mathrm{C}\right){ }^{\circ} \mathrm{K} \\
\text { Temperature, standard conditions }\left(+20{ }^{\circ} \mathrm{C}\right)^{\circ} \mathrm{K}\end{array}$ & 293 & 293 \\
\hline $\begin{array}{l}\text { Коэффициент сжимаемости системы, атм } \\
\text { System compressibility factor, } \text { atm }^{-1}\end{array}$ & $4,32 \cdot 10^{-4}$ & $1,95 \cdot 10^{-4}$ \\
\hline Пористость/Porosity & 0,13 & 0,135 \\
\hline $\begin{array}{l}\text { Дебит скважины до остановки, } \text { м }^{3} / \text { сут } \\
\text { Well rate before shut-in, } \mathrm{m}^{3} / \text { day }\end{array}$ & 137 & 11 \\
\hline $\begin{array}{l}\text { Экспертная оценка радиальной проницаемости } \\
\text { Expert of radial flow permeability }\end{array}$ & - & - \\
\hline
\end{tabular}
build-up curve)

Таблииа 2. Исходные данные и дополнительные априорные сведения по скважинам № 1, 2

Table 2. Initial and additional a priori data of wells no. 1,2 
Таблица 3. Результаты интерпретации КВД скважин № 1 и 2

Table 3. $\quad$ Results of pressure build-up curve interpretation of wells no. 1 and 2

\begin{tabular}{|c|c|c|c|c|c|c|}
\hline \multirow[b]{2}{*}{$\begin{array}{l}\text { КВД } \\
\text { Pressure } \\
\text { build-up } \\
\text { curve }\end{array}$} & \multirow[b]{2}{*}{$\begin{array}{l}\text { Метод } \\
\text { Method }\end{array}$} & \multirow[b]{2}{*}{$\begin{array}{l}\text { Сква- } \\
\text { жина } \\
\text { Well }\end{array}$} & \multirow[b]{2}{*}{$\begin{array}{c}\text { Время } \\
\text { Time }\end{array}$} & \multicolumn{3}{|c|}{$\begin{array}{c}\text { Оценки параметров пласта } \\
\text { Estimates of reservoir } \\
\text { parameters }\end{array}$} \\
\hline & & & & 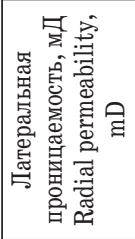 & 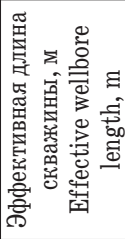 & 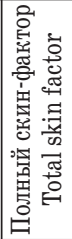 \\
\hline \multirow{4}{*}{$\begin{array}{c}\text { Полная } \\
\text { Total }\end{array}$} & \multirow{2}{*}{ Saphir } & 1 & 179 & 889 & 356 & $-5,93$ \\
\hline & & 2 & 366 & 0,67 & 119 & $-4,85$ \\
\hline & \multirow{2}{*}{$\mathrm{AИ}$} & 1 & 32 & 896 & 369 & $-5,86$ \\
\hline & & 2 & 80 & 0,60 & 115 & $-5,10$ \\
\hline \multirow{4}{*}{$\begin{array}{l}\text { Восстано- } \\
\text { вленная } \\
\text { Recovered }\end{array}$} & \multirow{2}{*}{ Saphir } & 1 & 179 & 801 & 399 & $-6,35$ \\
\hline & & 2 & 366 & 0,54 & 100 & $-5,10$ \\
\hline & \multirow{2}{*}{ АИ } & 1 & 10 & 911 & 367 & $-5,84$ \\
\hline & & 2 & 40 & 0,59 & 119 & $-5,11$ \\
\hline
\end{tabular}

В табл. 3 приведены оценки параметров пласта и скважины, полученные к моменту времени их стабилизации с использованием программы Saphir и методом адаптивной интерпретации. Эффективная длина горизонтального ствола определяется по формуле (10), где $\alpha_{2, \pi, n}^{*}-$ оценки параметра модели линейного потока. Оценки полного скин-фактора получены из модели позднего радиального потока по формуле (11), где $\alpha_{1}^{*}, \alpha_{2}^{*}-$ параметры модели позднего радиального потока.

$$
\begin{gathered}
L_{n}^{*}=\frac{C_{s} q B}{h \alpha_{2 \pi, n}^{*}} \sqrt{\frac{\mu}{\pi k_{r} C_{t}}} ; \\
S_{\text {общ }}^{*}=0.5\left(\frac{\alpha_{1}^{*}}{\alpha_{2}^{*}}-2.3 \log \left(\frac{2.25 k_{x y}}{m C_{t} \mu r_{c}^{2}}\right)\right) .
\end{gathered}
$$

Анализ результатов, приведенных на рис. 5, 6 и в табл. 3 , показывает, что адаптивный метод ин-

\section{СПИСОК ЛИТЕРАТУРЫ}

1. Развитие геофизического и гидродинамического мониторинга на этапе перехода к разработке объектов с трудноизвлекаемыми запасами нефти / В.Г. Мартынов, А.И. Ипатов, М.И. Кременецкий, Д.Н. Гуляев, В.М. Кричевский, В.В. Кокурина, С.И. Мельников // Нефтяное хозяйство. - 2014. - № 3. C. $106-109$.

2. Диагностика сложных трещин в коллекторах низкой проницаемости по результатам гидродинамических исследований / А.А. Вячистая, В.В. Кокурина, М.И. Кременецкий, Е.И. Гришина, Н.А. Морозовский // Каротажник. - 2017. - № 3. C. $38-61$.

3. Bourdet D., Ayoub J.A., Pirard Y.M. Use of pressure derivative in well test interpretation // Society of Petroleum Engineers. 1989. - № 12777. - P. 293-302.

4. Joshi S.D. Horizontal well technology. - Oklahoma: PenWell Publ. comp., 1991. - 381 p.

5. Хисамов Р.С., Сулейманов Э.И., Фархуллин Р.Г. Гидродинамические исследования скважин и методы обработки результатов измерений. - М.: ОАО «ВНИИОЭНГ», 2000. - 228 с. терпретации КВД горизонтальных скважин на основе прогнозирующих моделей позволяет обрабатывать короткие недовосстановленные КВД и определять параметры пласта и скважины, что существенно сокращает время простоя скважин. Для двух горизонтальных скважин необходимое время исследований при использовании программного комплекса Saphir составляет 545 ч. С помощью метода адаптивной интерпретации КВД достаточно 50 ч гидродинамических исследований скважин.

\section{Выводы}

Предложены модели и алгоритмы прогнозирования недовосстановленного участка КВД с использованием феноменологических моделей с переменными параметрами с учетом и корректировкой дополнительной информации о пластовом давлении.

На примере обработки данных гидродинамических исследований двух горизонтальных скважин Верхнеченского нефтяного месторождения методом адаптивной интерпретации показано, что разработанные модели и алгоритмы позволяют:

1) прогнозировать забойное давление на недовосстановленном участке КВД;

2) в процессе проведения исследований определять латеральную проницаемость, пластовое давление, скин-фактор, эффективную длину скважины и время завершения гидродинамических исследований в условиях отсутствия на кривой восстановления давления участка позднего радиального потока;

3) сократить время простоя скважин (применение метода адаптивной идентификации и интерпретации на прогнозирующих моделях для двух горизонтальных скважин позволяет сократить время простоя скважин в 10 раз с 545 до 50 ч).

6. Kuchuk F.J., Onur M., Hollaender F. Pressure transient formation and well testing: Convolution, Deconvolution and Nonline Estimation. - Amsterdam: Elsevier, Developments in Petroleum Science, 2010. - $389 \mathrm{p}$.

7. Kuchuk F.J. Well testing and interpretation for horizontal wells // Journal of Petroleum Technology. - January 1995. - V. 47. № 1. - P. 36-41.

8. Кременецкий М.И., Ипатов А.И., Гуляев Д.Н. Информационное обеспечение и технологии гидродинамического моделирования нефтяных и газовых скважин. - М.; Ижевск: Институт компьютерных исследований, 2012. - 896 с.

9. Сергеев В.Л., Донг Ван Хоанг. Адаптивная интерпретация гидродинамических исследований горизонтальных скважин с идентификацией псевдорадиального потока // Известия Томского политехнического университета. Инжиниринг георесурсов. - 2017. - Т. 328. - № 10. - С. 67-73.

10. Пантелеев А.В., Летова Т.А. Методы оптимизации в примерах и задачах. - СПб.: Изд-во «Лань», 2015. - 512 с.

11. Sergeev V.L., Dong Van Hoang. Adaptive identification and interpretation of pressure transient tests of horizontal wells: Chal- 
lenges and perspectives // Proc. IOP Conference Series: Earth and Environmental Science. - 2016. - V. 43. - № 1. - P. 1-6.

12. Хасанов М.М., Карачурин Н.А., Тяжев Е.А. Оценка извлекаемых запасов на основе феноменологических моделей // Вестник инженерного центра ЮКОС. - 2001. - № 2. - С. 3-7.

13. Мирзаджанзаде А.Х., Хасанов М.М., Бахтизин Р.Н. Моделирование процессов нефтегазодобычи. Нелинейность, неравновесность, неопределенность. - М.; Ижевск: Институт компьютерных исследований, 2005. - 368 с.

14. Соколов В.А. Эволюционные управления как феноменологические модели разработки нефтяных залежей // Нефтегазовое дело: электрон. науч. журн. - 2006. - № 2. - C. 1-36. URL: http://ogbus.ru/authors/SokolovVA/SokolovVA_1.pdf (дата обращения 20.01.2017).

15. Clark A.J., Lake L.W., Patzek T.W. Production forecasting with logistic growth models // Society of Petroleum Engineers. 2011. - № 144790. - P. 1-11.

16. Tsoularis A., Wallace J. Analysis of logistic growth models // Mathematical Biosciences. - 2002. - № 179. - P. 21-55.
17. Valko P.P., Lee W.J. A better way to forecast production from unconventional gas wells // Society of Petroleum Engineers. 2010. - № 134231. - P. 1-16.

18. Yousuf W., Blasingame T.A. New models for time-cumulative behavior of unconventional reservoir-diagnostic relations, production forecasting, and EUR methods / Unconventional resources technology conference. - 2016. - № 2461766. - P. 1-24.

19. Сергеев В.Л., Нгуен К.Х., Нгуен Т.Х.Ф. Адаптивная идентификация жизненного цикла систем методом интегрированных феноменологических моделей с переменными параметрами // Известия Томского политехнического университета. Инжиниринг георесурсов. - 2016. - Т. 327. - № 12. - С. 101-109.

20. Сергеев В.Л., Ву К.Д. Адаптивная интерпретации гидродинамических исследований с учетом влияния ствола скважины // Известия Томского политехнического университета. Инжиниринг георесурсов. - 2016. - Т. 327. - № 9. - С. 70-77.

Поступила 02.10.2018 г.

\section{Информация об авторах}

Сергеев В.Л., доктор технических наук, профессор отделения нефтегазового дела Инженерной школы природных ресурсов Национального исследовательского Томского политехнического университета.

Донг Ван Хоанг, аспирант отделения нефтегазового дела Инженерной школы природных ресурсов Национального исследовательского Томского политехнического университета.

Фам Динь Ан, магистр отделения нефтегазового дела Инженерной школы природных ресурсов Национального исследовательского Томского политехнического университета. 


\title{
ADAPTIVE INTERPRETATION OF THE RESULTS OF HORIZONTAL WELL PRODUCTION TESTING USING FORECASTING MODELS
}

\author{
Viktor L. Sergeev', \\ SergeevVL@ignd.tpu.ru \\ Dong Van Hoang', \\ hoang.tpu@gmail.com \\ Pham Dinh $\mathrm{An}^{1}$, \\ phaman.tpu@gmail.com \\ 1 National Research Tomsk Polytechnic University, \\ 30, Lenin Avenue, Tomsk, 634050, Russia.
}

The relevance of the research is caused by the need to interpret the results of testing horizontal well in the absence of the late-time radial flow on pressure build-up curves.

The main aim of the research is to develop models and algorithms for adaptive identification and interpretation of testing horizontal wells with forecasting the late-time radial flow regime of pressure build-up curves.

The methods. The authors have used the theoretical and practical developments in the field of adaptive interpretation of well testing, optimization methods, linear algebra, current research of system analysis, identification and adaptation of system. To solve the problems of identification and forecasting, the authors used evolutionary phenomenological models of pressure build-up curves with variable time-dependent parameters taking into account additional prior information on reservoir pressure. The pressure build-up curves were interpreted on the basis of the adaptive identification method. The problems were solved using the field data of testing horizontal wells of oil fields on the pressure build-up curves.

The results. The authors investigated potentialities of evolutionary phenomenological models with variable parameters for identifying and forecasting the pressure build-up curve in the absence of the late-time radial flow regime. The results of interpretation of testing horizontal well show that the developed models and algorithms of adaptive identification and interpretation allow: reliable enough prediction of bottomhole pressure that has not recovered on the pressure build-up curve; determination of lateral permeability, skin factor, effective well length and the well shut-in time during the well testing, significant reduction of the well shut-in time.

Key words:

Interpretation, identification, adaptation, well testing, pressure build-up curve, horizontal wells, I ow-permeability oil reservoir, phenomenological models, a prior information.

\section{REFERENCES}

1. Martynov V.G., Ipatov A.I., Kremenetskiy M.I., Gulyaev D.N., Krichevskiy V.M., Kokurina V.V., Melnikov S.I. Permanent reservoir monitoring by logging gages at the stage of tight oil recovery. Neftyanoe khozyaystvo, 2014, no. 1, pp. 106-109. In Rus.

2. Vyachistaya A.A., Kokurina B.B., Kremenetskiy M.I., Grishina E.I., Morozovskiy N.A. Diagnostic complex fructures in lowpermeability reservoir based on the results of pressure transient testing. Karotaznik, 2017, no. 3, pp. 38-61. In Rus.

3. Bourdet D., Ayoub J.A., Pirard Y.M. Use of pressure derivative in well test interpretation. Society of Petroleum Engineers, 1989, no. 12777 , pp. 293-302.

4. Joshi S.D. Horizontal well technology. Oklahoma, PenWell Publ. comp., 1991. $381 \mathrm{p}$.

5. Khisamov R.S., Suleymanov E.I., Farkhullin R.G. Gidrodina micheskie issledovaniya skvazhin i metody obrabotki rezultatov izmereny [Hydrodynamic studies of wells and methods of processing the results of measurements]. Moscow, VNIIOENG Publ., 2000.228 p.

6. Kuchuk F.J., Onur M., Hollaender F. Pressure transient formation and well testing: Convolution, Deconvolution and Nonline Estimation. Amsterdam, Elsevier, Developments in Petroleum Science, $2010.389 \mathrm{p}$.

7. Kuchuk F.J. Well testing and interpretation for horizontal wells. Journal of Petroleum Technology, January 1995, vol. 47, no. 1, pp. 36-41.

8. Kremenetsky M.I., Ipatov A.I., Gulyaev D.N. Informatsionoe obespechenie $i$ tekhnologii gidrodinamicheskogo modelorovaniya neftyanykh $i$ gazovykh zalezhey [Information and technology of hydrodynamic modeling of oil and gas deposits]. Moscow; Izhevsk, Institute of computer research Press, 2012. 896 p.

9. Sergeev V.L., Dong Van Hoang. Adaptive interpretation of pressure transient tests of horizontal wells with pseudoradial flow identification. Bulletin of the Tomsk Polytechnic University. Geo Assets Engineering, 2017, vol. 328, no. 10, pp. 67-73. In Rus.

10. Panteleev A.V., Letova T.A. Metody optimizatsii v primerakh $i$ zadachakh [0ptimization methods in examples and tasks]. St-Petersburg, Lan Publ., 2015. $512 \mathrm{p}$

11. Sergeev V.L., Dong Van Hoang. Adaptive identification and interpretation of pressure transient tests of horizontal wells: Challenges and perspectives. Proc. IOP Conference Series: Earth and Environmental Science, 2016, vol. 43, no. 1, pp. 1-6.

12. Khasanov M.M., Karachurin N.A., Tyazev E.A. Estimation of recoverable resources based on phenomenological models. Bulletin of Engineering Center YuKOS, 2001, no. 2, pp. 3-7. In Rus.

13. Mirzadzhanzade A.Kh., Khasanov M.M., Bakhtizin R.N. Modelirovanie protsessov neftegazodobychi, Nelineynost, Neravnovesnost, Neopredelennost [Modeling of oil and gas production processes, Nonlinearity, Nonequilibrium, Uncertainty]. Moscow; Izhevsk, Institute of Computer Science, 2005. 368 p.

14. Sokolov V.A. Evolyutsionnye uravneniya kak fenomenologicheskie modeli razrabotki neftyanukh zalezhey [Evolution equations as phenomenological models of development of oil fields]. Neftegazovoe delo, 2006, no. 2, p. 1-36. Available at: http://ogbus.ru/authors/SokolovVA/SokolovVA 1 (accessed at 20 January 2017). 
15. Clark A.J., Lake L.W., Patzek T.W. Production forecasting with logistic growth models. Society of Petroleum Engineers, 2011, no. 144790 , pp. 1-11.

16. Tsoularis A., Wallace J. Analysis of logistic growth models. Mathematical Biosciences, 2002, no. 179, pp. 21-55.

17. Valko P.P., Lee W.J. A better way to forecast production from unconventional gas wells. Society of Petroleum Engineers, 2010, no. 134231, pp. 1-16.

18. Yousuf W., Blasingame T.A. New models for time-cumulative behavior of unconventional reservoir-diagnostic relations, production forecasting, and EUR methods. Unconventional resources technology conference, 2016, no. 2461766, pp. 1-24.
19. Sergeev V.L., Nguyen Q. Huy, Nguyen T.H. Phuong. Adaptive identification of system lifecycle by the method of integrated phenomenological models with variable parameters. Bulletin of the Tomsk Polytechnic University. Geo assets Engineering, 2016, vol. 327, no. 12, pp. 101-109. In Rus.

20. Sergeev V.L., Vu Quang Duc. Adaptive interpretation of the results of hydrodynamic research considering a wellbore storage. Bulletin of the Tomsk Polytechnic University. Geo assets Engineering, 2016, vol. 327, no. 9, pp. 70-77. In Rus.

Received: 2 October 2018.

\section{Information about the authors}

Viktor L. Sergeev, Dr. Sc., professor, National Research Tomsk Polytechnic University.

Dong Van Hoang, postgraduate, National Research Tomsk Polytechnic University.

Pham Dinh An, master, National Research Tomsk Polytechnic University. 